\title{
The C-Reactive Protein Response in Beta-Hemolytic Streptococcal Infections
}

\author{
Felicia Trofin $^{1,2}$, Luminita Smaranda Iancu ${ }^{1}$, EV Nastase ${ }^{* 1,2}$, Ioana Alina Harja Alexa ${ }^{1,2}$ and Olivia Simona \\ Dorneanu ${ }^{1,2}$ \\ ${ }^{1}$ University of Medicine and Pharmacy "Grigore T Popa" Iasi, Romania \\ ${ }^{2}$ Clinical Hospital of Infectious Diseases "Sf Parascheva" Iasi, Romania
}

*Corresponding author: EV Nastase, University of Medicine and Pharmacy

Received Date: November 12, 2019

"Grigore T. Popa" Iasi, Romania.

Published Date: November 19, 2019

\section{Abstract}

Background: C-reactive protein (CRP) is an acute-phase serum protein that activates the complement. It is secreted by the liver in response to inflammatory cytokines. CRP levels increase rapidly after tissue damage in response to inflammation and infection and decrease with the resolution of the condition.

Material and method: We analyzed 230 patients that had positive cultures of the pharyngeal exudate for group A, Cor $\mathrm{G} \beta$-hemolytic streptococci, admitted to the Clinical Hospital of Infectious Diseases "Sf. Parascheva" Iasi between 1.07.2018-31.12.2018, which we have corroborated with inflammation markers (CRP, leucocyte count, fibrinogen) and with clinical diagnosis.

Results: Only 155 (67.4\%) patients were confirmed with streptococcal angina or scarlet fever. $72.2 \%$ of the patients have been diagnosed with group A streptococci (GAS) infection, $14.2 \%$ with group C streptococci (GCS) and 13.5\% with group G streptococci (GGS) infection. 75 patients were healthy carriers of $\beta$-hemolytic streptococci: GAS was isolated from $23.8 \%$, GCS from $60.7 \%$ and GGS from $22.2 \%$ patients. In $90.9 \%$ of GCS infections CRP values were above $50 \mathrm{mg} / \mathrm{L}$, while $38.4 \%$ and, respectively, $23.8 \%$ of those infected with GAS or GGS have such values. In only $52.3 \%$ GGS patients CRP level was above $5 \mathrm{mg} / \mathrm{L}$.

Conclusion: 1. The most common streptococcal pharynx infections were GAS ones, followed by GCS and GGS. 2. CRP values in patients with GCS infection are more often above $50 \mathrm{mg} / \mathrm{L}$ than in those with GAS or GGS infections. 3. Pharyngeal GCS carriage is the most common of all pathogenic $\beta$-hemolytic streptococci. 4. The inflammatory response to GGS is low.

Keywords: C-reactive protein; Beta hemolytic streptococci; Infection

Abbreviations: CRP: C-reactive Protein, GAS: Group A Streptococcus, GCS: Group C Streptococcus, GGS: Group G Streptococcus

\section{Introduction}

C-reactive protein (CRP) was introduced into medical practice in the 1990s as a rapid orientation test [1]. Some factors that may influence CRP levels are age, sex, weight, cholesterol level, blood pressure, smoking status [1]. The native CRP is composed of 5 identical subunits bound by non-covalent bonds, placed around a central pore, in the characteristic form of "lectin fold", as a betafolded sheet [2]. It irreversibly dissociates in inflammation into 5 monomers, the monomeric CRP. The native CRP activates the classical complement pathway and it induces apoptosis and phagocytosis. The monomeric CRP plays an important role in chemotaxis and in directing leukocytes to the inflammatory area, delaying apoptosis. The human CRP gene can be found at $1 \mathrm{q} 23.2$ on the long arm of chromosome 1 , and, to date, there have been no allelic variations or genetic deficiencies discovered for this gene although some polymorphisms have been identified [1]. CRP synthesis starts in response to pro-inflammatory cytokines, particularly IL6, IL1 and TNF alpha [3].

Kingsley and Jones [4] state that in the case of infections, CRP increases in response to the cytokines IL6 and IL1 and has a stable rate of decomposition. It is mainly synthesized by hepatocytes but can also be synthesized by smooth muscle tissue [5], macrophages [6], endothelial cells [7], lymphocytes and adipocytes [8]. It will bind to microbial polysaccharides and damaged cell ligands by activating the complement. CRP levels increase rapidly, up to 1000fold after tissue injury as a systemic response to inflammation or infection and decrease exponentially with condition resolution [9]. 
CRP provides information about the inflammatory reaction and it helps to manage the antibiotic therapy [10]. Black, et al. [11] specify that CRP is not only a marker of inflammation and infection, but also plays a protective role against infectious agents by activating the complement system. Kingsley and Jones [4] state that CRP can be used in the differentiation between colonization and infection, but it cannot help to distinguish between types of infection. Huang, et al. [12] assert that the determination of CRP values decreased the rate of antibiotic therapy in lower respiratory tract infections.

Cals and Weert [13] highlighted an association between high CRP values and bacterial infections. Acute pharyngitis is one of the most common complaints that a physician encounters in the ambulatory care setting. Typically, the incidence peaks during childhood and in adolescents. Although there are a large number of visits each year for pharyngitis, the majority of these cases are viral and are self-limiting. However, group A streptococcus (GAS) is the most common bacterial etiology for acute pharyngitis. The prevalence of streptococcal pharyngeal infections in non-A group streptococcus is constantly increasing. The rapid antigen detection test limits doctors to the determination of GAS [14]. As the rapid diagnosis of infectious diseases is an important practical problem for the case and the doctor, with an epidemiological, social, family, biological and economic impact, we attempted to establish the CRP response in different type beta-hemolytic streptococcal infections.

\section{Material and Methods}

The main purpose consists in conducting a retrospective study of microbiologically confirmed cases with pharyngeal $\beta$-hemolytic streptococci, by assessing the rate and the mean values of CRP according to the pathogen agent and establishing the status of infectious or colonizing agent. We analyzed 230 consecutive patients admitted to the Clinical Hospital of Infectious Diseases "Sf. Parascheva" Iasi between 1.07.2018-31.12.2018, which follow the inclusion criteria. Inclusion criteria: positive culture of the pharyngeal exudate for group A, C or G $\beta$-hemolytic streptococci; CRP level determined for the patient. Exclusion criteria: culture negative for $\beta$-hemolytic streptococci; incomplete laboratory data for the patient. The examination protocol included CRP values,

which we have corroborated with inflammation markers such as white blood cell count and differential, fibrinogen and with clinical diagnosis.

One pharyngeal swab per patient was inoculated on Columbia Blood Agar (Bio maxima, Poland) and incubated at $37^{\circ} \mathrm{C}$ in an $5 \%$ carbon dioxide atmosphere for 48 hours. A culture was considered positive for beta-hemolytic streptococcus with a growth of any number of large beta-hemolytic colonies, gram-positive staining with streptococcal morphology, and a negative catalase test. Serogrouping of beta-hemolytic streptococci was performed with the Streptococcal Grouping Kit (Oxoid, UK). The culture was considered negative when beta-hemolytic colonies of group A, C, G streptococci were absent after 48 hours of incubation. CRP was quantified using RX Imola Chemistry Analyzer (Furuno, Japan).

\section{Result}

A total of 230 patients were enrolled. Only 155 (67.4\%) patients were confirmed with streptococcal angina or scarlet fever, based on clinical presentation and inflammatory syndrome. The rest of 75 patients, without inflammatory signs were considered healthy carriers of $\beta$-hemolytic streptococci: GAS was isolated from 35 patients (23.8\% of GAS positive pharyngeal exudate), respectively, group C streptococci (GCS) from 34 patients (60.7\% of GCS positive pharyngeal exudate) and group G streptococci (GGS) from 6 patients (22.2\% of GGS positive pharyngeal exudate). GAS was identified in 61 males (41.5\%) and in 86 females (58.5\%), GCS in 20 males (35.7\%) and 36 females (64.3\%), GGS in 16 males (59.3\%) and 11 females (40.7\%). GAS and GCS were more frequent in females, but GGS was most common in males.

55 of the patients $(23.9 \%$ of the total number of patients) were diagnosed with scarlet fever; 52 of them (94.6\%) was confirmed with GAS, 1 (1.8\%) with GCS and 2 (3.6\%) with GGS. 100 patients $(43.5 \%)$ had the diagnosis of angina; 60 of them $(60 \%)$ was confirmed with GAS, 21 (21\%) with GCS and 19 (19\%) with GGS. The rest of them $(32.6 \%)$ were colonized with beta-hemolytic streptococcus. The age of the patients ranged from 1 to 86 years. No significant differences were found in age between patients (Table 1).

Table 1: Number of patients per investigated parameter and clinical diagnosis.

\begin{tabular}{|c|c|c|c|c|c|c|c|c|}
\hline \multirow[t]{2}{*}{ Strep Group } & \multirow[t]{2}{*}{ Total Patients (no) } & \multicolumn{5}{|c|}{$\mathrm{CRP}(\mathrm{mg} / \mathrm{dL})$} & \multicolumn{2}{|c|}{ Diagnosis } \\
\hline & & $<5$ & $5-50$ & $50-100$ & $100-150$ & $>150$ & Scarlet fever & Angina \\
\hline $\mathrm{A}$ & 112 & 15 & 54 & 29 & 6 & 8 & 52 & 60 \\
\hline $\mathrm{C}$ & 22 & 2 & 0 & 7 & 6 & 7 & 1 & 21 \\
\hline G & 21 & 10 & 6 & 3 & 1 & 1 & 2 & 19 \\
\hline
\end{tabular}

\section{Discussion}

Only $67 \%$ of the patients from whom we isolated beta-hemolytic streptococci were infected. The most frequent carrier state was for GCS (Figure 1). The most frequent infection was produced by GAS, present in 112 cases (72.2\% of infected patients), followed by GCS with $14.2 \%$ (22 patients) and GGS with $13.5 \%$ (21 patients)
(Figure 2). Infection with groups GCS was associated with higher CRP concentrations (mean $134 \mathrm{mg} / \mathrm{L}$ ) compared with those with GAS infection (mean $55 \mathrm{mg} / \mathrm{L}$ ) and GGS infection (mean $32 \mathrm{mg} / \mathrm{L}$ ). In $90.9 \%$ of GCS infections CRP values were above $50 \mathrm{mg} / \mathrm{L}$, while $38.4 \%$ and, $23.8 \%$, respectively, of those infected with GAS or GGS had such values (Figures 3,4,5). The highest CRP concentration 
was observed among patients with group C streptococcus infection (Figure 4). We found that in GGS infected patients' inflammatory syndrome was not important; in only 52.3\% GGS patients CRP level was above $5 \mathrm{mg} / \mathrm{L}$ (Figure 5).

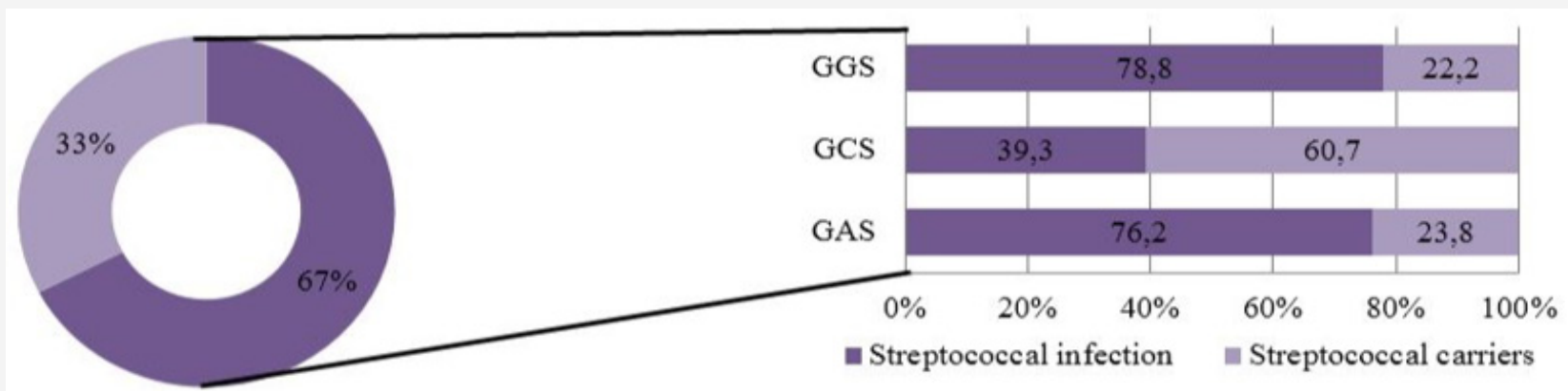

Figure 1: Beta-hemolytic Streptococci infection and pharyngeal carriage, with a differential in GAS, GCS and GGS.

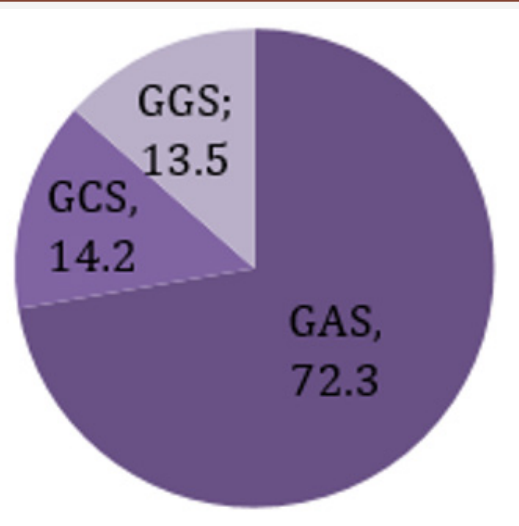

Figure 2: Incidence of Beta-hemolytic Streptococci Infections.

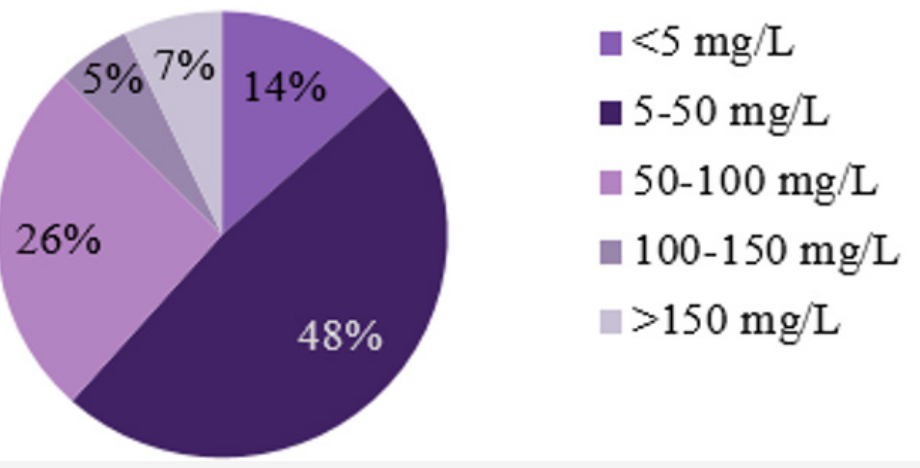

Figure 3: CRP values in GAS.

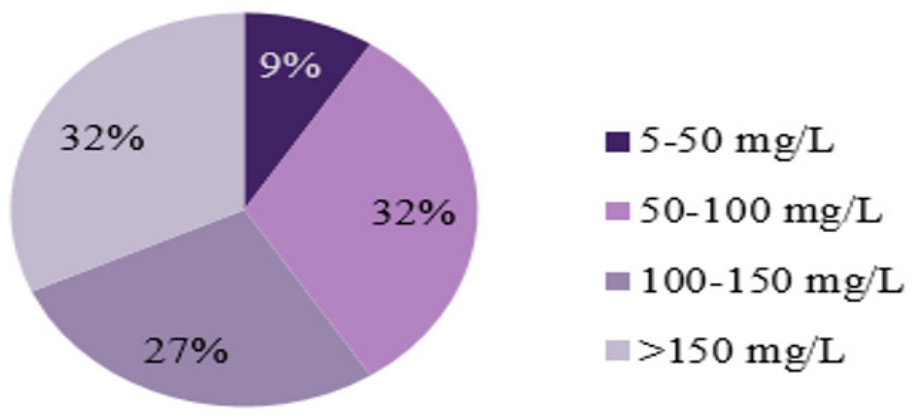

Figure 4: CRP value in GCS. 


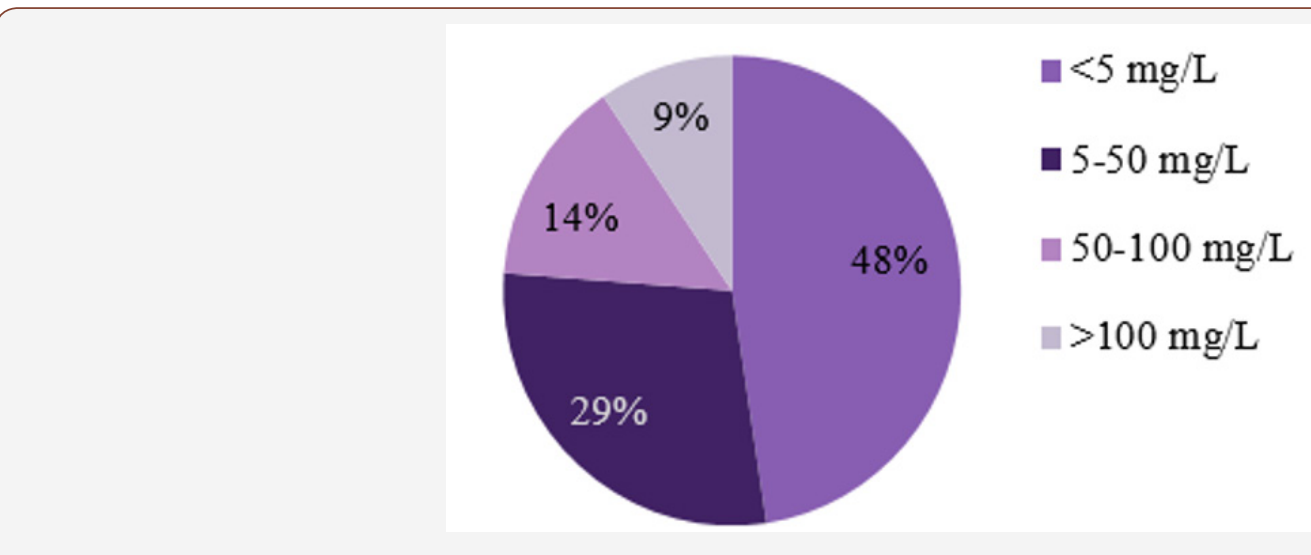

Figure 5: CRP value in GGS.

This is one of the few studies that emphasized the role of CRP as a rapid orientation test in the diagnosis of $\mathrm{A}, \mathrm{C}$ or $\mathrm{G}$ streptococcal infection. CRP provides information about the inflammatory response and can be used as a guidance in managing the antibiotic therapy. CRP can make the difference between a streptococcal infection and a streptococcus healthy carrier with angina caused by a viral infection, by highlighting the presence of the inflammatory reaction. Our analysis awares that group C and G streptococci should be considered as throat pathogens along with GAS. The frequency of patients with GCS and GGS infection is about one fifth of the patients with GAS, as it has also been shown in other studies [15-17], we may consider that GCS and GGS are frequent causes of streptococcal infections. Few previous studies have analysed the variation of CRP in group A, C and G streptococcal infections. Our findings are in agreement with Calviño, et al. [18] from the Institute of Primary Care and Public Health, Cardiff University School of Medicine, that analysed the association between CRP rapid test and GAS infection. They observed an increased frequency of GAS infections compared to GCS and GGS, but a higher median CRP value $(49.9 \mathrm{mg} / \mathrm{L})$ in patients with GCS and GGS infections, with the peak value recorded in GCS patients.

Furthermore, our results are similar with the results obtained by Lindbæk, et al. [17]. They have looked into the clinical symptoms and signs in sore throat patients with large colony variant $\beta$-haemolytic streptococci groups $\mathrm{C}$ or $\mathrm{G}$ versus group $\mathrm{A}$. They concluded that CRP levels above $25 \mathrm{mg} / \mathrm{L}$ were significantly associated with presence of streptococci group C or G. They also stated that group C and G streptococci should be considered as throat pathogens in line with GAS. Hjortdahl and Melbye [19] examinated if on-site testing enhances the diagnosis of streptococcal pharyngitis in adults. They obtained a mean CRP value of $50.4 \mathrm{mg} / \mathrm{L}$ in their GAS patients. Our mean CRP value in GAS infected patients of $55 \mathrm{mg} / \mathrm{L}$ was similar to their findings.

Melbye, et al. [20] observed the daily reduction in C-reactive protein values in group-A streptococcal pharyngitis treated with antibiotics. They examined daily eleven adult patients with sore throat and confirmed GAS infection. Their average CRP value was of $100.3 \mathrm{mg} / \mathrm{L}$, higher than our result (55mg/L). This difference may be caused by our significantly bigger number of GAS patients (147 patients compared with 11 patients).

There are some authors that suggest that CRP testing is overused $[21,22]$. Our results reveal that CRP is useful for differentiating GAS infection from GCS and GGS ones and from other etiologies that do not require antibiotic therapy. Nowadays, the diagnosis of sore throat is based on microbiological identification. The culture of the pharyngeal exudates is the gold standard used when evaluating the etiology of the infection. Rapid detection of increased CRP values in sore throat patients can differentiate the bacterial from the viral angina, thus being able to increase the number of correctly treated patients. One strengh of this study is that we have considered a statistically significant number of patients.

\section{Conclusion}

1. The most common streptococcal pharyngeal infections were produced by GAS, followed by GCS and GGS.

2. CRP values in patients with GCS infection are more often above $50 \mathrm{mg} / \mathrm{L}$ than in those with GAS or GGS infections.

3. Pharyngeal GCS carriage is the most common of all pathogenic $\beta$-hemolytic streptococci.

4. The inflammatory response to GGS is low.

\section{Acknowledgement}

None.

\section{Conflict of Interest}

No conflict of interest.

\section{References}

1. Hage FG, Szalai AJ (2007) C-reactive protein gene polymorphisms, C-reactive protein blood levels and cardiovascular disease risk. J Am Coll Cardiol 50(12): 1115-1122.

2. Eisenhardt SU, Thiele JR, Bannasch H, Stark GB, Peter K (2009) C-reactive protein: how conformational changes influence inflammatory properties. Cell Cycle 8(23): 3885-3892.

3. Zhang D, Sun M, Samols D, Kushner I (1996) STAT3 participates in transcriptional activation of the C-reactive protein gene by interleukin-6. J Biol Chem 271(16): 9503-9509. 
4. Kingsley A, Jones V (2008) Diagnosing wound infection: the use of C-reactive protein. Wounds UK 4(4): 32-46.

5. Calabró P, Willerson JT, Yeh ET (2003) Inflammatory cytokines stimulated C-reactive protein production by human coronary artery smooth muscle cells. Circulation 108(16): 1930-1932.

6. Devaraj S, Singh U, Jialal I (2009) The evolving role of C-reactive protein in atherothrombosis. Clin Chem 55(2): 229-238.

7. Pasceri V, Willerson JT, Yeh ET (2000) Direct proinflammatory effect of C-reactive protein on human endothelial cells. Circulation 102(18): 2165-2168.

8. Calabró P, Chang DW, Willerson JT, Yeh ET (2005) Release of C-reactive protein in response to inflammatory cytokines by human adipocytes: linking obesity to vascular inflammation. J Am CollCardiol 46(6): 11121113.

9. Thompson D, Pepys MB, Wood SP (1999) The physiological structure of human C-reactive protein and its complex with phosphocholine. Structure 7(2): 169-177.

10. André M, Vernby A, Odenholt I, Lundborg CS, Axelsson I, et al. (2008) Diagnosis-prescribing surveys in 2000, 2002 and 2005 in Swedish general practice: consultations, diagnosis, diagnostics and treatment choices. Scand J Infect Dis 40: 648-654.

11. Black S, Kushner I, Samols D (2004) C-reactive protein. J Biol Chem 279(47): 48487-48490.

12. Huang Y, Chen R, Wu T, Wei X, Guo A (2013) Association between point-of-care CRP testing and antibiotic prescribing in respiratory tract infections: a systematic review and meta-analysis of primary care studies. Br J Gen Pract 63(616): e787-e794.

13. Cals J, Henk Van Weert (2003) Point-of-care tests in general practice: Hope or hype? Eur J Gen Pract 19(4): 251-256.
14. Banerjee S and Ford C (2018) Rapid Tests for the Diagnosis of Group A Streptococcal Infection: A Review of Diagnostic Test Accuracy, Clinical Utility, Safety, and Cost-Effectiveness. CADTH Rapid Response Report: Peer Reviewed Summary with Critical Appraisal, Canada.

15. Meland E, Digranes A, Skjaerven R (1993) Assessment of clinical features predicting streptococcal pharyngitis. Scand J Infect Dis 25(2): 177-183.

16. Zwart S, Sachs AP, Ruijs GJ, Gubbels JW, Hoes AW, et al. (2000) Penicillin for acute sore throat: randomized double-blind trial of seven days versus three days treatment or placebo in adults. BMJ 320(7228): 150-154.

17. Lindbæk M, Høiby EA, Lermark G, Steinsholt IM, Hjortdahl P (2005) Clinical symptoms and signs in sore throat patients with large colony variant beta-haemolytic streptococci groups $\mathrm{C}$ or $\mathrm{G}$ versus group $\mathrm{A}$. $\mathrm{Br}$ J Gen Pract 55: 615-619.

18. Calviño O, Llor C, Gómez F, González E, Sarvisé C, Hernández S (2014 May-Jun) Association between C-reactive protein rapid test and group A streptococcus infection in acute pharyngitis. J Am Board Fam Med 27(3): 424-426.

19. Hjortdahl P, Melbye H (1994) Does near-to-patient testing contribute to the diagnosis of streptococcal pharyngitis in adults? Scand J Prim Health Care 12: 70-76.

20. Melbye H, Bjørkheim MK, Leinan $T$ (2002) Daily reduction in C-reactive protein values, symptoms, signs and temperature in group-A streptococcal pharyngitis treated with antibiotics. Scand J Clin Lab Invest 62(7): 521-525.

21. Engström S, Mölstad S, Lindström K, Nilsson G, Borgquist L (2004) Excessive use of rapid tests in respiratory tract infections in Swedish primary health care. Scand J Infect Dis 36: 213-218.

22. Sproston NR, Ashworth JJ (2018) Role of C-Reactive Protein at Sites of Inflammation and Infection. Front Immunol 9: 754. 